\title{
Retracing Quantum Steps
}

Two authors of a feature article in Europhysics News would like the chance to supplement their list of references

After the publication of Quantum Conductance: a Step-by-Step Guide in the last issue of $E N$, it has been drawn to our attention that we neglected to mention two recent publications which also describe how electromagnetic relays can be used to produce quantised conductance steps. These are: H.Yasuda and A. Sakai Phys. Rev. B 56 (1997) 1069 and, especially, K. Hansen, E. Laegsgaard, I. Stensgaard and F. Besenbacher Phys. Rev. B 56 (1997) 2208 which includes many useful details of the experimental procedure. We wish to apologize to these authors for our omission.

Though our article was not intended to be a review, there are some other key references which deserve to be mentioned: $\mathrm{C}$. J. Miller, J. M. van Ruitenbeck and L. J. de Jongh Phys. Rev. Lett. 69 (1992) 140 for the first clear observation of quantum steps in mechanically controllable break junctions; and M. Brandbyge et al, Phys. Rev. B 52 (1995) 8499 and J. M. Krans et al, Nature 375 (1995) 767 for the use of conductance histograms and detailed discussion of quantum conductance results.

It is also necessary to point out that the simple Landauer formalism may only be useful as a starting point for the understanding of conductance at point contacts. Even though it has been very successful in describing the conductance in metals like $\mathrm{Na}$, in particular the disappearance of some conductance plateaus because of degenerate modes, its application to other metals is still controversial. For monovalent metals the first conductance plateau, which arises from a contact geometry of a single atom connecting the two electrodes, is usually understood to be quantum in nature. However, higher order peaks are more difficult to interpret since the processes taking place during the contact breaking are complex: tunnelling contributions are important and steps in conductance may arise from atomic rearrangements so that the conductance peaks are usually found over a large smooth background. The case of nonmonovalent metals is even more complex, see E. Sheer et al, Phys. Rev. Lett. 78 (1997) 3535 and J.C. Cuevas et al, Phys. Rev. Lett. 8 o (1998) 1066.

The 'quantisation of conductance' at point contacts has not been fully explored, is not yet fully understood, and is likely to remain a topic of further study and discussion for some time to come.

\section{Frederic Ott and James Lunney}

Trinity College, Dublin

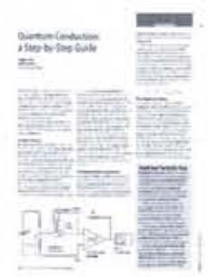

The article by Frederic $0 \mathrm{tt}$ and James Lunney appeared in the last issue of Europhysics News (291)

\section{CALL FOR PROPOSALS}

\section{HIGH FIELD MAGNET LABORATORY UNIVERSITY of NIJMEGEN}

The High Field Magnet Laboratory at the University of Nijmegen is an international user facility where high continuous fields (up to 30 tesla) together with the necessary experimental infrastructure (like $\mathrm{mK}$ temperatures, visible and far infrared spectroscopy, measurements of thermodynamic quantities etc.) are available for research, Scientists of EU countries are intitled to apply under the "TMR-Access to LargeScale Facilities". Proposals will be reviewed by an International Program Advisory Committee, and qualified projects will be given full access and experimental support free of charge, for all research meant for publication in the op literature.

Information about available equipment, proposal forms and procedures is available from M.A. Lange, tel +31-24-3652087, fax +31-24-3652314, email marthag@sci.kun.nl, or also at the Institute Home Page http://www.sci.kun.nl/

\section{CECAM}

\section{Centre Européen de Calcul Atomique et Moléculaire}

\section{Ecole Normale Supérieure de Lyon, Aile LR5} 46 Allée d'Italie, 69364 Lyon Cedex 07, France

CECAM (European Center for Atomic and Molecular Calculations) is searching its future Director, for a four year term starting on 1st September 1998.

CECAM is an organization which is funded by several institutions from European countries (Belgium, France, Greece, Italy, Netherlands, Switzerland and UK). Its aim is to promote large scale classical and quantum simulations applied to Condensed Matter, Chemistry and Biology. The activities of CECAM involve the hosting of a few researchers and the organizing of workshops. More information about these activities can be found on the Web site: http://www.cecam.fr/.

The new Director should be a senior scientist with the level of a Laboratory Leader or University Professor, having a large experience in one or several fields such as Condensed Matter, Chemistry, Statistical Mechanics, Numerical Simulations. The nature and level of appointment of the position will depend on the qualification of the candidate. Applications should be sent to F. Perrot, at the address below, before March 31, 1998. The final selection will take place in Lyon (France) on 14 May 1998.

F. Perrot

CEA/BIII/DCSA/BatE, Boîte Postale n 12, 91680 Bruyères-le Châtel, France

Tel: 33.01 .69 .26 .42 .08 . Fax: 33.01 .69 .26 .70 .90 